\title{
Review: Atopic Dermatitis - Exposome, Microbiome, Psyche,and Evolution
}

\author{
Julian Penev', Milla Balabanova ${ }^{2}$,IlkoBakardzhiev ${ }^{3}$ \\ ${ }^{1}$ Varna Dept. Infections and Skin Diseases, MU-Varna, Bulgaria \\ ${ }^{2}$ VFU ChernorizetzHrabar SDK Psychology, Varna, Bulgaria \\ ${ }^{3}$ College of Medicine, MU- Varna, Bulgaria
}

Received: January 11, 2021; Accepted: January 24, 2021; Published: February 02, 2021

*Corresponding author: MillaBalabanova, VFUChernorizetzHrabar SDK Psychology, Varna, Bulgaria, Tel. No: +359882050299;. E-mail: millybalabanova@gmail.com

\section{Abstract}

Atopic Dermatitis (AD) is probably the most common type of eczema. The quality of life assessment reveals a number of negative consequences for patients with $\mathrm{AD}$ and their families. $\mathrm{AD}$ is associated with high levels of stress, stigma, social withdrawal, anxiety, depression, and even suicide ideation. Atopic dermatitis is a multisystem inflammatory disease with a multifactorial, and still controversial etiology. Complex interactions between genetic, psychoneuroimmune and endocrine factors, microbiome, exposome and environment, mental and social factors, diet and lifestyle are proposed. AD is difficult to treat, has a chronic course with peaks of exacerbation and pronounced manifestation of mental factors. Such complexity requires deeper understanding of mechanisms behind AD. We consider a possible psychogenic component in diseaseexacerbation, andoffera different point of view onrelationships of human body with the colonizing microbiomein the context of AD etiology and pathogenesis. These relationships are interpreted in evolutionary terms in an attempt to shed some light on mechanisms of chronic and pathological skin processes.

Keywords: Atopic Dermatitis; Microbiome; Exposome;ObsessiveCompulsive Disorder

Atopic dermatitis is probably the most common type of eczema. It affects a total of about $30 \%$ of children and adults $[1,2]$.Its external symptoms are characterized by sebostatic, ichthyosiform and xerotic skin. AD is a multisystem inflammatory disease with a multifactorial etiology. Complex interactions between genetic, psychoneuroimmune and endocrine factors, microbiome, mental and social factors, diet and lifestyle and other exposomefactors respectively, are taken into consideration. Such complexity and taken evidence that AD is difficult to treat, has a chronic course with peaks of exacerbation, with no lasting response to therapies,as well ascertain manifestation of mental factors call foranalyzingthe disease from different perspectives. In our opinion, relationship of human organism and its colonizing microbiome should be reviewedto assess mechanisms that are responsible for forming a healthy barrier that separates organism from exposome.
Atopic dermatitis is examined in a wide range of research areas due to its strong impact on psychosocial functioning, and hence on subjective quality of life. Dry, itchy, red, often cracked, scaly (ichthyosiform) skin inevitably causes discomfort. Sufferers do complain of low self-esteem due to bothcompromised appearance and condition of their skin. They may feel anxious and insecure about their social performance.AD has a strong impact on psychosocial functioning, and therefore on subjective quality of life. Quality of life is further impaired by lack of sleep due to affected areas' severe itch. A myriad of negative consequences such as: low self-esteem,unfavorablephysical appearance'selfperception, social interactions apprehension,restriction of certain desired activities, sleep deprivation, disease' financial burden, overuseof various cosmetics, remedies, and medications in attempts to relieve itching etc., can result inhigh levels of stress.Alternate relapses and remissions are additional stressors, as patients are anxiously waiting for the unpleasant symptoms to worsen again. Since skin changes can engage face, body, and the capillary worries related to physical appearance and lack of physical attractiveness, in particular are typical for AD sufferers. Desquamations and itchy plaques around the nose, mouth, neck, and eye area often create definite concerns related to facial aesthetics and attractiveness. This is especially true for those affected by the disease in adolescence. It is then that appearance related self-esteem is most sensitive and vulnerable to attitudes, and others' comments. In general, detrimental effects of skin diseases on mental health and different aspects of of life should not be underestimated[3].Although $\mathrm{AD}$ can occur at any age it often begins in early childhood as it attracts attention of various fields' specialists - dermatologists, pediatricians, psychologists, allergologists, etc., due to its early manifestation and diverse comorbidity [4]. Assessment of associated quality of life proves the many adverse consequences for patients and their families (e.g. certain choice of professional realization, certain choice of clothing, avoidance of socialization, refusal of certain sports and other activities, etc.) [5]. AD is associated with high levels of stress, stigma, social withdrawal, anxiety, depression and even suicide ideation among those affected [6-9].Sufferers may have high levels of anxiety when starting a close emotional relationship and may tend to avoid affective attachment [8]. 
Certain factors indicate that $\mathrm{AD}$ is a neurotic register' disorder. Patients with AD may also develop secondary mental problems due to the appearance of their skin. Personality traits and/or psychiatric comorbidity can lead to secondary mental disorders [6,10]. Psychoemotional factors are considered in $\mathrm{AD}$ etiopathogenesis.Skininteracts directly with external environment, and ambiguously responds to its stimuli. Skin reflects, reproduces, and responds to emotional stimuliin social interactions,and mental and physical state. Course of many skin diseases, incl. ADis affected by stress,anxiety and various mental events, according to scientific literature [11]. AD is considered a typical psychosomatic disorder provided its cyclical course reflects external manifestation of emotions, i.e. subjective emotional interpretation of events, interpersonal interactions and relationships, psychotraumatic situations.Emotional factors can also cause and intensify the behavior of itching and scratching [6]. Stress and anxiety are considered in AD etiopathogenesis, and as triggers of immune disorders. It is possible for stress to modulate course of atopic dermatitis through neuroendocrine pathways and induction of TH2 [12-15]phenotype. Neuroendocrine mediators - incl. Adrenocorticotropin, $\beta$-endorphin and cortisol are produced in response to stress, and as a result the increase in endogenous glucocorticoids can disrupt barrier function of the skin, making it vulnerable to inflammatory diseases such as atopic dermatitis [6]. It is known, severe and even obsessive scratching on the affected areas of the body is responsible for much of the suffering of those with $\mathrm{AD}$, and traces of such behavior are easily seen with the appearance of eczematous lesions on areas of the body that the hands can reach [16]. Many patients with AD describe such behavior just as "habitual scratching". However, self-excoriation behavior, along with other manifestations of self-aggressive behavior (compulsive repeated bathing throughout the day, constant skin exfoliation, antibacterial and other detergents, body odor nauseaetc., indicate the presence of psychogenic componentandmay provide some explanation of mechanisms behind disease exacerbation. According to our observations, most patients with $\mathrm{AD}$ describe their itching sensation as burning, annoying, unpleasant and even painful, and their emotional state as feeling anxious or even angry. The "need" to scratch the areas and experienced pleasure of scratching gradually increase the stronger the itching.In our practice,we have also observedtendency of autoexcoriation duringcertain rites performed throughout the day: bodywashing with surfactants,antibacterial and detergent products, along withrepetitive skin exfoliating

Actions of self-excoriation and repeated washing whereasskin epidermal integrity is deliberately damaged provide an occasion to justify such actions as autoaggressive or obsessive-compulsive, and OCD related. OCD is not a personality disorder. It is a neurotic disorder - i.e. the individualsare pretty much aware of their actions and also that they should not do soyet,they simply cannot do otherwise. Providedthat patients are informed by their physician that self-excoriation behavior is a powerful factor in prolonging symptoms it is assumed they realize they should not perform it. However, they admit that they cannot refrain from doing so.In fact, certain actions performed and described by such patients constitute rituals to alleviate suffering and achieve mental comfort. These hyperhygienic ritualsare obligatory as performed in series of actions that are regularly repeated during the day in a specific way. In informal conversation, such AD patients admit that they cannot give up performing their own hyperhygienic customs. No matter how hard they aretrying to overcome them, they fail for the growing tension is alleviated only by performing the ritual.

It is this ritual that indicates a connection with obsessivecompulsive pathology. Compulsive excessive bodywashing is performed in an attempt to wash away dirt and germs, to relieve severe stress and itching, to improve appearance of affected skin areas. Such patients have a history of fear of germs and sweating, nausea from their body odor, and constant use of antibacterial agents and large amounts of laundry detergent to achieve best possible hygiene of their clothes. Bathing several times per day with fragranced surfactants aims in particular, according to patients' own wordsis to completely eliminate any body odor;scrubs are supposed to remove all impurities from the skin along with the "dead cells" and the "microbes"; to achieve "ephemeral purity", reduce anxiety, and bothersome itching. Such actions are also intended to hopefully improve overall skin look for negative emotions are experienced, and especially whenfacial zones are affected.Above described behaviors provide basis for consideringof such actions as being autoaggressive (pathomimic), and possibly related to dysmorphophobia. Therefore, possible links between $\mathrm{AD}$ and Obsessive-Compulsive Disorder (OCD) should closelybe investigated.

A very important factor for a healthy skin,actively studied in recent years is the condition of skin microbiome. Skin is an organ that makes contact with the outside world. In evolutionary terms, it performs basic functions related to sensory perception of the world around,and person's own body boundaries, sexual attractiveness, libido, etc. It determines communication through touch and tactile emotional experiences. Healthy skin featuresare interpreted as visual markers of reproductive potential. A very important healthy skin factor is the condition of skin microbiome. Its biological sense is whether the skin is not inhabited by immunetolerant symbiotic microbial fauna it will be attacked by pathogenic and resistant to therapeutic effects microorganisms. Blood immunity has a memory of skin microbiome, and whether an inflammatory reaction caused by microorganisms (injuries, abrasions, scratches) occurs,the immune system may solve the problem quickly. Provided that skin microbiomebalance is being disturbed (by detergents, antibiotics, corticosteroids, retinoids, etc.), antibiotic-resistant and aggressive bacterial strains known to cause nosocomial infections add to the inflammatory process.They deepen the inflammatory process and recovery slows down. Pathological processes can be exacerbated and become resistant to treatment.There is evidence that exposure of children in early childhood to certain microorganisms (intestinal flora and helminthic parasites) protects against allergic diseases contributing to the development of the immune system $[17,18]$ while the lack of such exposure leads to defects in the establishment of immune tolerance $[17,19-21]$ and immune 
autoaggression. Epidemiological studies support the protective role of helminths against $\mathrm{AD}$ [21],and that children raised with pets have a lower risk of atopic dermatitis [21,22].

Exposome domains are categorized as nonspecific (human and natural factors), specific (ultraviolet radiation, allergens, humidity, etc.), and internal (microbiome and interaction with host cells) [12,23].Identified are external environmental factors contributing to the pathogenesis of AD - dust particles, organic compounds, allergens, etc. $[9,24,25]$.These factors are likely to provoke immune responses in atopic skin [12]. External factors contributing to disruption of skin permeability barrier are also frequency of washing, use of surfactants, as well as self-scratching and exfoliation behavior further leading to deterioration of epidermal permeability.In our opinion, current appearance of human skin has changed relatively recently with the use of clothing and the reduction of body and facial hair. This change has led to a change ofpilosebaceus unit, which has also affected human skinmicrobiome.Different animal species have different microbiome structures related to whether they are predators, herbivores, nocturnal/diurnal animals, habitation, etc.

In human evolution, along with the emergence of specific anthropogenic factors, such as hygiene and clothing the human microbiome has formed. Microbiome is probably still in the process of evolutionary improvement given the brief evolution of Homo sapiens. The imperfect microbiome is probably the cause of most of the dermatosis in Homo sapiens. During the last century hygienic habits become a major factor strongly influencing skin microbiome condition. With the addition of newly synthesized chemical compounds in cosmetics and hygiene products, thesehabits have become an indisputable exposome element. Exposome has been actively studied in the last decade. Until recently, symbiotic colonization of our skin by microorganisms and their importance to skin condition were accepted by the civilized world almost as a heresy. Achieved understanding of the role of the microbiome and its relationship to skin health has strengthened scientific interest in this issue and has begun to change, albeit very timidly, scientific and public opinion.Nowadays, there aremany commercial advertisements claiming aboutcosmetics and hygiene products being gentle on skin microbiome. Cosmetics with prebiotic action are also availablefor sale. This is not surprising at all, since surfactants wash away lipid protection, as well as part of keratinocyteswhich have a definite protective function in evolutionary terms. Overuse of shampoos, shower gels, soaps in AD violates skin lipid barrier which becomes more vulnerable to exposome factors. Urea, a natural hydrating factor is washed away, removed is skin sebum, skin microbiome'environment and balance are disturbed. Hyperhygienic actions are largely result of family upbringing and imitation, and are driven by marketing strategies. As a fine example,all bacteria arepresented in advertisements as ugly and repulsive cartoon creatures that should be destroyed, incl. and from the skin with a variety of antibacterial products and surfactants.

Mechanisms that are directly related to healthy skin are tuned within the evolutionarily formed symbiotic relationship between the human genome and that of the microbiome. In our opinion, skin microbiome and the epidermis are a system, covering the entire body. This system can be likened to a protective "spacesuit" suit against the exposome domains. It contains dead keratinocytes, whose mummified structure includes melanosomal particles which serve as a natural photo protection; the sebum and urea secreted by the sebaceous and sweat glands retain the moisture so necessary for the germinative cells of the epidermis and melanocytes. This "spacesuit" is saturated with sensors and bacterial nanorobots for the entire world around. The suit has a direct connection with organism's functions of reproduction. It releases pheromone substances that guarantee finding the love partner. The system is isolated from the blood immune system through the dermoepidermal barrier, whileskin immunityinteracts in a specific way with microbial agents and has developed a certain immune tolerance to them.

This system is typical to all biological structures (species) and its evolutionary improvement ensures successful adaptation of animal species.Use of detergents that wash away and disrupt the structure of the epidermis-microbiome inevitably triggers development of pathological processes with varying degree of severity (depending on individual characteristics, habitat and social exposure, etc.). Dermatology describes these pathological processes as such with an inflammatory-immune component in their pathogenesis (atopic dermatitis, seborrheic dermatitis, rosacea),while others are potentiated as infectious dermatoses (mycoses, some skin viruses, andzoonoses).

Many microorganisms are accused of being related to skin diseases when in fact they are part of the microbiome. The role of propilobacterum acne, for example, is greatly exaggerated in the pathogenesis of acne [26,27].It is believed to be the cause of acne and gets attacked by antibiotics which further worsen the condition of skin microbiome. Such treatment is more palliative than definitive for it attacks only the infectious component of the disease, and this component is most often caused by antibiotic-resistant pathological strains. In other words, the treatment is ineffective and essentially pointless. Preparation of a microbiogram (i.e. a study of the presence and percentage distribution of microbiomesymbiotes) is essential to assess the quality of the barrier and the condition of the skin. We believe the key to successful treatment of atopic dermatitis is an integrated approach. A multidisciplinary team may be needed (a dermatologist, psychiatrist, neurologist, clinical psychologist) to provide high quality and effective patient care.It is important to carefully take a complete history of the patient. Questioning the patient is a conscious process on the part of the doctor which incorporates attentive and active listening, asking explanatory questions,patient's mental state observation and assessment of emotional reactions,as well as information about the patient's life, family history and bad habits, and integration of received information. 


\section{Conclusion}

Treatment of atopic dermatitis requires a revised approach. Relationships of human organism with the inhabiting skin microbiomeare to be reexaminedto betterunderstand mechanisms of interaction that are crucial to form and maintain a healthy barrier (healthy skin) that separatethe body from the exposome. Complex mechanisms and factors that are being responsible for a homeostatically healthy skin are in constant dynamics. In our opinion, a specific component may dominate the etiopathogenesis of $\mathrm{AD}$ of each individual. It is important to look for the possible leading component that unlocks and maintains, along with other factors the symptoms for the particular patient. When there is no pronounced psychogenic component, application of therapeutic minimalism may be an appropriate solution - limiting all detergents,skinexfoliating agents and cosmetics,as well as protection from physical factors (UV radiation, cold, wind, high temperatures, etc.).

\section{References}

1. Weidinger S, Beck LA, Bieber T, Kabashima K, Irvine AD. Atopic dermatitis. Nat Rev Dis Prim. 2018;4(1):1.

2. Barbarot S, Auziere S, Gadkari A, Girolomoni G, Puig L, EL Simpson, et al. Epidemiology of atopic dermatitis in adults: Results from an international survey. Allergy. 2018;73(6):1284-1293. doi:10.1111/ all.13401

3. Bewley A. The neglected psychological effects of skin disease. BMJ. 2017;358:j3208.

4. Trashlieva M. Atopic eczema. Information for general practitioners, medical staff, parents and patients. Peychinski Publishing House, Pleven. 1999

5. LifschitzC. The Impact of Atopic Dermatitis on Quality of Life. Ann Nutr Metab. 2015;66(suppl 1):34-40 doi: 10.1159/000370226

6. Senra MS, Wollenberg A. Psychodermatological aspects of atopic dermatitis. British Journal of Dermatology. 2014.

7. Jafferany M. Psychodermatology: a guide to understanding common psychocutaneous disorders. Prim Care Companion J Clin Psychiatry 2007;9:203-213. doi:10.4088/pcc.v09n0306

8. Maksimovic N, Zaric M, Bjelica S, Marinkovic JE, Jankovic S. Psychosomatic factors of atopic dermatitis exacerbation. Int J Dermatol.2018;57(9):1080-1084. doi:10.1111/ ijd.14076

9. Powers CE, McShane DB, Gilligan PH, Burkhart CN, Morrell DS. Microbiome and pediatric atopic dermatitis. J Dermatol. 2015;42(12):1137-1142.

10. Hassan I, Keen MA, Bhat YJ, Latif I. Psychodermatoses in children. Indian J PaediatrDermatol. 2019;20(4):306-314.
11. Picardi A, Abeni D, Melchi CF, Puddu P, Pasquini P. Psychiatric morbidity in dermatological outpatients: An issue to be recognized. $\mathrm{Br}$ J Dermatol. 2000;143:983991.

12. Stefanovic N, Flohr C, Irvine A. The exposome in atopic dermatitis. Allergy. 2019.

13. Elias PM, Hatano Y, Williams ML. Basis for the barrier abnormality in atopic dermatitis: Outside-inside-outside pathogenic mechanisms. J Allergy Clin Immunol. 2008;121(6):1337-1343. doi:10.1016/j. jaci.2008.01.022

14. Arndt J, Smith N, Tausk F. Stress and atopic dermatitis. Curr Allergy Asthma Rep.2008;8:312-317. doi:10.1007/s11882-008-0050-6

15. Lin T-K, Zhong L, Santiago J. Association between Stress and the HPA Axis in the Atopic Dermatitis. Int J Mol Sci. 2017;18(10):2131. doi:10.3390/ijms18102131

16. KamideR.Atopic Dermatitis: Psychological Care. JMAJ.2002;45(11):490-495.

17. Scudellari M. News Feature: Cleaning up the hygiene hypothesis. Proceedings of the National Academy of Sciences. 2017;114(7):14331436.

18. StiemsmaL, Reynolds L, Turvey S, Finlay B. The hygiene hypothesis: current perspectives and future therapies. Immuno Targets and Therapy. 2015;143.

19. Silva A, Gabriel M, Oliveira A, Cerni A, Zottich U, Manuela B. The hygiene hypothesis at a glance: Early exposures, immune mechanism and novel therapies. Acta Tropica.2018;188:16-26. doi:10.1016/j. actatropica.2018.08.032

20.Bieber T. Atopic dermatitis. The New England Journal of Medicine. 2008,4;358(14):1483-1494.

21. Flohr C, Mann J. New insides into the epidemiology of childhood atopic dermatitis. Allergy. 2014;69(1):3-16. Doi:10.1111/all.12270

22. Pelucchi C, Galeone C, Bach JF, La Vecchia C, Chatenoud L. Pet exposure and risk of atopic dermatitis at the pediatric age: a meta-analysis of birth cohort studies. The Journal of Allergy and Clinical Immunology. 2013;132(3):616-622.e7.

23. Tsakok T, Woolf R, Smith CH, Weidinger S, Flohr C. Atopic dermatitis: the skin barrier and beyond. Br J Dermatol. 2018.

24. Ahn K. The role of air pollutants in atopic dermatitis. J Allergy Clin Immunol. 2014;134(5):993-999. doi:10.1016/j.jaci.2014.09.023

25. Fu T, Keiser E, Linos E, Rotatori RM, Sainani K, Lingala B, et al. Eczema and sensitization to common allergens in the United States: A multiethnic, population-based study. Pediatr Dermatol. 2014;31(1):21-26. 
26. Paller AS, Kong HH, Seed P, Naik S, Scharschmidt TC, Gallo RL, et al. The Microbiome in Atopic Dermatitis, Journal of Allergy and Clinical Immunology. 2018. doi:10.1016/j.jaci.2018.11.015.
27. PicardiA,Mazzotti E, Pasquini P. Prevalence and correlates of suicidal ideation among patients with skin disease. J Am AcadDermatol. 2006;54:420-426. 